\title{
Multiple Impacts of Debris on a Vertical Obstacle
}

\author{
Nils Goseberg, Technische Universität Braunschweig, contact@nilsgoseberg.de \\ Jacob Stolle, University of Ottawa, jstol065@uottawa.ca \\ loan Nistor, University of Ottawa, inistor@uottawa.ca
}

\section{BACKGROUND}

Past tsunami events have caused extreme damage in coastal regions. Examples are the Indian Ocean Tsunami in 2004 and the Tohuku Tsunami in 2011. These extreme natural disasters brought into light the devastating nature which tsunami-induced inundation might inflict when propagating on-land. As a result of eye-witness reports and extensive media coverage, a wealth of evidence showing multi-phase fluid motion entraining a solid phase consisting of entrained debris, ranging from sediment grains to large vessels became available. In this context, debris impacts have been linked to major infrastructural damage (Yeh et al. 2012). This observation resulted in increased research emphasis on tsunami-driven debris impact. It also initiated the inclusion of first debris impact force equations in existing building codes such as FEMA (P-646 2012) and ASCE (Standard 7-16 Chapter 6). There are however still a lot of uncertainties on factors influencing tsunami-driven debris impact. Besides the random, probabilistic nature of debris entrainment, advection by the entraining flow and the uncertainty related to impact points, no guidance exists as to how multiple impacts of debris can be accounted for. In addition, little focus was directed to impact forces on nonrigid structures which investigated here for the first time. OBJECTIVES

This research is part of a comprehensive study initiated by the researchers at the Leibniz Universität Hannover, Germany and the University of Ottawa, Canada, with the overall goal to aim at multi-impact debris loading on vertical structures. The objectives guiding this specific research are:

- Investigating hydrodynamic and impact loads exerted on a non-rigid vertical structure

- Examining the probability of multiple impact events and what influences them

- Providing guidance on the structural responses in comparison with hydrodynamic base loads.

EXPERIMENTAL SETUP

The experiments were conducted in a hydraulic flume at the University of Ottawa (Canada). The facility is equipped with a swing gate which can impound water on its upstream side with a head of up to $50 \mathrm{~cm}$. Its rapid opening was achieved by a manually-operated release mechanism and additional dead weight attached to the gate at the end of a cantilever. The debris, modelled as 1:40 scaled-down shipping containers, were placed downstream of the gate, allowing sufficient distance for the wave to fully develop. A comprehensive test program was conducted which included also single debris testing (Derschum et al., 2017) as well as debris arrangements with up to 12 shipping containers, either placed normal or perpendicular to the approach flow. The distance between individual debris was set to $0.06 \mathrm{~m}$ throughout the tests. Depending on the number of debris involved, either one or two layers of containers were used. Two hydrodynamic forcing conditions $(0.20 \mathrm{~m}$ and 0.40 $\mathrm{m}$ impoundment depths) were examined with a minimum of 10 repetitions. The debris motion was tracked using an novel optical tracking algorithm developed by the authors (Stolle et al., 2016). Additionally, a smart debris system as described by Goseberg et al. (2016) was installed inside the shipping container models to allow for redundant tracking. A vertical structure was placed downstream of the impoundment acting as an obstacle to the debris entrained in the dam-break flow. The structure was installed onto a six-axis dynamometer and its base mount was designed to allow for some flexibility so as to ensure a certain level of non-rigidity of the structure.

RESULTS AND DISCUSSION

The impact test with multiple debris entrained by the dambreak wave resulted in the debris agglomerating throughout the acceleration process, essentially acting as a single mass. As a result, the debris accelerated slower as compared to single debris, falling thus behind the wave tip and therefore reaching a lower maximum velocity. However, the debris agglomeration often broke up when approaching the structure. This was a result of the stagnation zone upstream of the obstacle. Secondly, random impacts of the debris on the structure were observed. A number of impact scenarios which can be linked to the measured force response of the vertical structure were therefore identified. Figure 1 depicts an example of the time-history of flow-wise debris-induced forces recorded during a double impact event. Strong oscillations of the structure with a peak force response in exceedance of the prescriptions currently implemented by the ASCE7-16 standard were observed. While the second impact event showed lower force response, it might still be relevant in cases where the first impact could have resulted in partial failure. Onset of building collapse or failure initiation of structural members could be explained by subsequent impact events, previously overlooked by tsunami engineering guidelines.

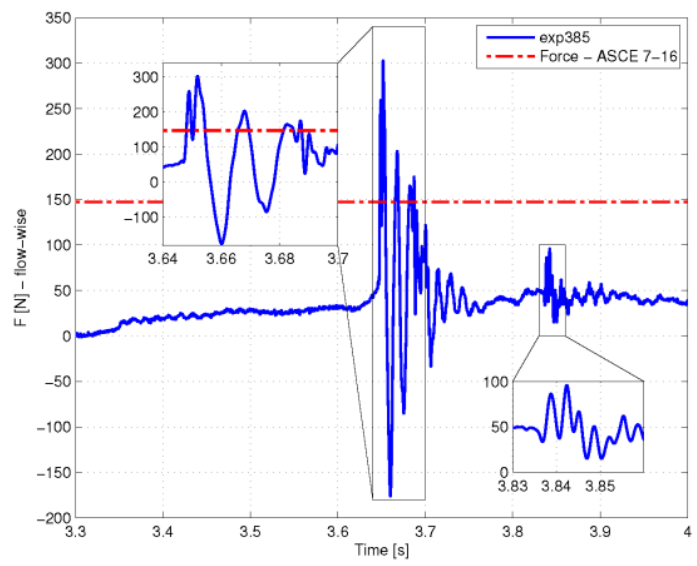

Figure 1 - Force response of a structure exposed to flow and a multiple impact event.

CONCLUSIONS

Research results discussed herein will identify structural response as a result of multiple impacts. It will provide preliminary suggestions as to when multiple impacts should be considered in the design process of buildings in a hazard zone. Statistical information on impact will also be investigated.

REFERENCES

Due to space limitations, references mentioned herein were not provided. 